\title{
The Effect of An Aqueous Electricidal Solution on General Well Being
}

\author{
Leonard Sonnenschein ${ }^{1 *}$, Tiberious Etyang ${ }^{2}$, Rutu Shah ${ }^{3}$, Ruth Frischer ${ }^{4}$ and Glen Rein ${ }^{4}$ \\ ${ }^{1}$ The Sonnenschein Institute, USA \\ ${ }^{2}$ eNSPIRE Africa, Kenya \\ ${ }^{3}$ Case Western Reserve University, USA \\ ${ }^{4}$ International Health Scientist, USA
}

*Corresponding author: Leonard Sonnenschein, The Sonnenschein Institute, 6617 NW 24th Ave., Boca Raton, FL 33496, (314) 609-2798, USA.

To Cite This Article: Leonard Sonnenschein, Tiberious Etyang, Rutu Shah, Ruth Frischer, Glen Rein. The Effect of An Aqueous Electricidal Solution on General Well Being. Am J Biomed Sci \& Res. 2021 - 13(4). AJBSR.MS.ID.001899. DOI: 10.34297/AJBSR.2021.13.001899.

Received: 畊 July 16, 2021; Published: 眥 July 23, 2021

\begin{abstract}

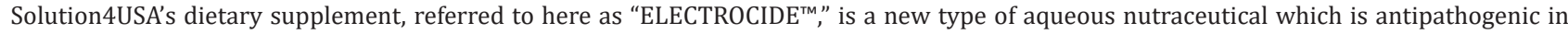
nature due to pathogen specific electrical charge properties. Thousands of people have been treated with this product when delivered through an existing network of medical professionals validating its efficacy and safety. Using FDA guidelines, "ELECTROCIDE ${ }^{\text {TM" }}$ was evaluated for wellness benefits as a potential investigational drug under medical supervision using 52 volunteers (Group1: 15 and Group2: 37) in an institutional setting (The Lovelady Center (TLC) in Birmingham, Alabama) using quantitative measurements of vital signs along with two qualitative self-reported questionnaires. The results indicate a highly significant improvement in vital signs and a significant reduction in various physiological and emotional conditions.
\end{abstract}

At the start of the study, the average temperature and oxygen values were subnormal, indicating poor health status, whereas, by the end of the study, vital signs were all normal. During the study, a cohort study (proof of concept) was conducted with 14 individuals, in the TLC general population, who were identified and diagnosed as COVID-19 positive. After 6 days (on average) of "ELECTROCIDE ${ }^{\text {TM" }}$ administration, a shift of their COVID-19 status switched to negative, and a complete reversal of symptoms were observed several times faster than predicted by the CDC [1]. Of particular interest were oxygen levels because a previous in-vitro pilot study indicated that dissolved oxygen levels doubled with "ELECTROCIDE ${ }^{\text {TM" }}$ -It is postulated that this is an important mechanism promoting an immediate sense of wellness.

Keywords: Electrical Charge; Dietary Supplement; Mineral Solution; Oxygen; Electricidal; Wellbeing; Antipathogen; Inflammation; CoVID-19; Wellness; Electrocide; Bacterial; Fungi; Viruses

Abbreviations: TLC: The Lovelady Center, EM: Electromagnetic, SB: Small Body, MB: Medium Body, LB: Large Body

\section{Introduction}

Electroceuticals are typically bioelectronic signaling devices that deliver electrical energy to the body transcutaneously using electrodes [2]. One can also use patches [3], fabrics [4], biofilms [5], and hydrogels [2] utilizing metallic salts and ions to generate current voltages. Electroceuticals are bactericidal since DC currents as low as 10 micro-amp in combination with electrically generated ions (Ag, $\mathrm{Cu}$, and $\mathrm{Zn}$ ) have been reported to kill bacterial [6], fungi [7], and viruses [4] in as little as 2 days. Micronized minerals and trace minerals have been shown to have broad-spectrum anti-pathogenic properties [8,9]. Scanning electron microscope images show pathogens being fragmented by the electrical discharge between the negatively charged pathogens and the positively charged surface of the active minerals $[10,11]$. It has been proposed that such "iontophoretic killing" of pathogens is mediated by a positive zeta potential generated from a negatively charged RNA envelope inside a positively charged capsid shell [11]. This proposed mechanism of action has recently been demonstrated using an electroceutical 
fabric that lowers the coronavirus's zeta potential, resulting in destabilization of its electro-kinetic properties eradicating its infectivity upon contact with the fabric [4].

In addition to having toxic effects on pathogenicmicroorganisms, bacteria and viruses, electroceuticals have therapeutic effects [12]. They have been shown to increase the bioavailability of dietary supplements $[9,13]$, enhance wound healing [14], have an analgesic action [15], and are used to treat venous leg ulcers [16]. Furthermore, since electroceuticals can target neural electrical circuits and modulate action potentials [17], they can also produce neural-based therapeutic effects. The aqueous electricidal solution used in the current study has another property that could also account for its clinical efficacy-it is hypotonic (Sonnenschein, patent pending). Hypotonic solutions have been used for osmoregulation and extracellular osmotic manipulation resulting in enhanced membrane transport of sodium via activation of Na-K ATPase [18]. This approach has been used to regulate cerebral edema [19], control intraocular pressure in the cornea [20], release glutamate from astrocytes [21], and cause hemolysis in red blood cells [22]. In addition, the aqueous electroceutical used in the current study is also a supersaturated oxygen solution. Hyperoxygenation is used clinically to modulate cognitive function [23], regeneration [24], and pulmonary circulation [25]. In addition, oxygenated water has been used to enhance athletic performance [26], treat diabetics [27], stimulate the immune system [28], accelerate detoxification [29], and increase oxygen availability [30].

\section{Methodology}

\section{Recruitment of Participants}

Informed consent was obtained through a release form and a medical history form from each participant, to ensure understanding of potential risks and benefits of consuming the product. All subjects, including the COVID-19 individuals, filled out a daily online survey for 3 days prior to "ELECTROCIDE ${ }^{\text {TM" }}$ administration that served as control measurements. The treatment groups continued to report the beneficial effects 1 hour after administration for 7 days. Throughout the study, every subject's blood was checked daily before and after using the "ELECTROCIDE ${ }^{\mathrm{TM}}$." Near the end of the study, any changes experienced from the initial marked conditions were documented as a percentage change from beginning to end, along with any additional comments. According to The Lovelady Center, it was against their policies to include a placebo group. Therefore, the participants in the first 3 days of the study prior to the administration of "ELECTROCIDE ${ }^{\text {TM" }}$ served as a control group.

\section{The Wellness Study Protocol}

A one-ounce bottle of $1 \mathrm{~mL}$ of "ELECTROCIDE ${ }^{\text {TM" }}$ concentrate was added, and the container was filled with tap water to yield 1-ounce net fluid. The bottles were labeled with product information and a mixing reminder to activate the suspension properly. They were also tagged for each participant to be refilled and reused throughout the study. When necessary, additional blood chemistry or other lab work was undertaken with such data to become part of the study, including COVID-19 Testing.

\section{Participants}

There were 15 participants in Group 1 (1-4 days of product administration), while 37 participated in Group 2 (5-7 days of product administration). The average age of Group 1 was 39.2 years and 41.3 years for Group 2. Participants in Group 1 averaged 2.5 out of 3 in body type $(\mathrm{SB}\{$ Small Body $\}=1 ; \mathrm{MB}\{$ Medium Body $\}=2$; LB $\{$ Large Body $\}=3$ ) while those in Group 2 averaged 2.2 out of 3 in body type. On average, $64 \%$ of Group 1 reported either smoking or vaping, while $76 \%$ in Group 2 reported smoking or vaping. Participants in Group 1 used an average of 2.7 doses, while those in Group 2 used an average of 6.0 doses. All personal information was kept confidential.

\section{Statistical Analyses}

Data were analyzed using the Student's T-test in a paired and non-paired format based on data using a two-tailed approach, since data can be bi-directional. $\mathrm{P}$ values where $\mathrm{p}<0.05^{*}$ is considered significant, $\mathrm{p}<0.01^{* *}$ is considered very significant, and $\mathrm{p}<0.001^{* * *}$ is considered extremely significant.

\section{COVID-19 Cohort Study}

During the general wellness study, 14 individuals in the general population of The Lovelady Center were noted to have tested positive for COVID-19, with associated symptoms. A cohort "proof of principal" study, comprised of these individuals, was added to the wellness study. However, vital signs and other observations were not performed in this group due to limited access to the infirmary where they were quarantined. However, notes regarding symptoms and the disappearance of symptoms were performed, along with

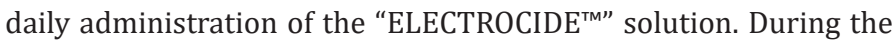
wellness study, none of the individuals participating in the wellness study at TLC became ill.

\section{Results}

\section{Medical History}

Initial Annotated Data were compared to last day's data as Percentage Improvement. Each value presented above represents the percent improvement in their symptomology by comparing their initial entry symptoms with their symptoms on day 7. Raw values for each time were determined using 1-10 numerical scale. Group 1 received 1-4 servings total and Group 2 received 5-7 servings total. All control data represents the average subjective evaluations (scale 0-10) for each subject during the 3-day control period. The overall control averages are compared with the average 
subjective evaluations 1 hour after taking the product on day 4 . For each parameter, the participants were asked to rank their daily health conditions. These conditions were rated 0 to 10 . If the parameter was noted to be -, then 0 would be the optimal numerical value, and 10 would be the worst condition. If the parameter were noted to be + , then 0 would be the worst condition, and 10 would be optimal (Table1-2). The data indicate a highly significant change in the symptomology at day 4 when the entire population (Group
1 and Group 2) were evaluated. Only stomachache and diarrhea issues were not significantly changed. The results indicate a statistically significant increase in both body temperature and blood oxygen levels towards the end of the study. At the start of the study, the average temperature and oxygen values were subnormal, indicating their poor health status, whereas, by the end of the study, their vital signs were normal (Table3-4).

\begin{tabular}{|c|c|c|c|c|c|}
\hline Test Group (Decrease \%) & Group 1 & Group 2 & Test Group (Increase \%) & Group 1 & Group 2 \\
\hline Stomach Problems & 50 & $100^{*}$ & Focus & 66.7 & $76.7^{*}$ \\
\hline Back pain & 30 & $83.3^{*}$ & Sleep & 100 & $97.0^{*}$ \\
\hline Leg/ knee pain & 50 & $82.2^{*}$ & Change in Energy & 100 & $96.8^{*}$ \\
\hline Numbness/Arm pain & 34 & $92.3^{*}$ & & & \\
\hline Arthritis & 33.1 & $75.0^{*}$ & & & \\
\hline Heart Problems & 30 & $100^{*}$ & & & \\
\hline Depression / Anxiety & 27.5 & $82.9 *$ & & & \\
\hline Asthma & 25 & $84.5^{*}$ & & & \\
\hline Lung/Breathing Problems & 14 & $91.8^{*}$ & & & \\
\hline Headache & 0 & $91.7^{*}$ & & & \\
\hline Neck pain & 0 & $83.3^{*}$ & & & \\
\hline \multicolumn{6}{|c|}{ *Indicates data has been shown to be statistically significant to $p<0.05$ probability } \\
\hline \multicolumn{6}{|c|}{$\begin{array}{l}\text { Each value presented above represents the percent improvement in their symptomology by comparing their initial entry symptoms with their } \\
\text { symptoms on day } 7 \text {. Raw values for each time were determined using 1-10 numerical scale. Group } 1 \text { received } 1-4 \text { servings total and Group } 2 \\
\text { received 5-7 servings total. }\end{array}$} \\
\hline
\end{tabular}

\begin{tabular}{|c|c|c|c|c|}
\hline \multirow{2}{*}{ Study Parameters } & \multicolumn{4}{|c|}{ Statistics } \\
\hline & Mean-All Control $(n=219)$ & Mean-Treat Day $4(n=32)$ & t-value & Net change: Treat \\
\hline Happy and Content & $6.7 \pm 0.29$ & $8.4 \pm 0.49^{* * *}$ & -4.282 & 1.7 \\
\hline Depressed & $4.1 \pm 0.54$ & $1.6 \pm 0.99 * * *$ & 4.847 & -2.5 \\
\hline Anxious & $5.1 \pm 0.61$ & $2.1 \pm 1.43^{* * *}$ & 5.403 & -3 \\
\hline Headache & $2.8 \pm 0.59$ & $1.3 \pm 1.25^{* *}$ & 2.773 & -1.5 \\
\hline Throat Issues & $1.1 \pm 0.32$ & $0 \pm 0.01^{* *}$ & 2.656 & -1.1 \\
\hline Trouble Breathing or Shortness of Breath & $2.5 \pm 0.65$ & $0.2 \pm 0.04^{* * *}$ & 4.301 & -2.3 \\
\hline Cough a lot & $1.8 \pm 0.41$ & $0.3 \pm 0.17^{* * *}$ & 3.487 & -1.5 \\
\hline Congested or having a Runny Nose & $2 \pm 0.43$ & $0.3 \pm 0.19 * * *$ & 3.782 & -1.7 \\
\hline Feel Nauseous & $1 \pm 0.3$ & $0.2 \pm 0.16^{*}$ & 2.133 & -0.8 \\
\hline Stomachache & $1 \pm 0.29$ & $0.4 \pm 0.34$ & 1.364 & -0.6 \\
\hline Diarrhea & $0.71 \pm 0.21$ & $0.2 \pm 0.13$ & 1.536 & -0.51 \\
\hline Back Pain & $4.33 \pm 0.71$ & $1.6 \pm 1.16^{* * *}$ & 4.577 & -2.73 \\
\hline Muscle Pain & $4.26 \pm 0.74$ & $1.8 \pm 1.34^{* * *}$ & 4.038 & -2.46 \\
\hline Able to Think Clearly & $7.17 \pm 0.52$ & $8.9 \pm 0.47^{* * *}$ & -3.465 & 1.73 \\
\hline Energy Level & $4.94 \pm 0.28$ & $6.4 \pm 1.06^{* * *}$ & -3.719 & 1.46 \\
\hline Feel Sleepy, Tired, or Fatigued & $5.51 \pm 0.43$ & $2.7 \pm 1.38^{* * *}$ & 5.876 & -2.81 \\
\hline \multicolumn{5}{|c|}{ *Significant, ${ }^{* *}$ Very Significant, ${ }^{* * *}$ extremely significant } \\
\hline \multicolumn{5}{|c|}{$\begin{array}{l}\text { All control data represents the average subjective evaluations (scale } 0-10 \text { ) for each subject during the } 3 \text {-day control period. The overall contro } \\
\text { averages are compared with the average subjective evaluations } 1 \text { hour after taking the product on day } 4 \text {. For each parameter, the participants were } \\
\text { asked to rank their daily health conditions. These conditions were rated } 0 \text { to } 10 \text {. If the parameter was noted to be }- \text {, then } 0 \text { would be the optima } \\
\text { numerical value, and } 10 \text { would be the worst condition. If the parameter were noted to be }+ \text {, then } 0 \text { would be the worst condition, and } 10 \text { would be } \\
\text { optimal. }\end{array}$} \\
\hline
\end{tabular}


Table 3: Temperature and Oxygen Vital Signs-Statistical Comparison.

\begin{tabular}{|c|c|c|c|c|}
\hline Statistics & Mean-Day 1 (n=25) & Mean-Day 6 (n=35) & t-value & p-Value \\
\hline Temperature & $96.6 \pm 0.48$ & $98 \pm 0.35^{* * *}$ & -5.24 & $<0.001$ \\
\hline Oxygen & $97.3 \pm 0.28$ & $98.3 \pm 0.30^{* *}$ & -2.65 & $<0.01$ \\
\hline
\end{tabular}

Each value represents the average measurement of their vital signs for each of the subjects prior to day 1 product consumption and 1 hour after day 6 product consumption. Day 1 acts as a control because the product had not been taken.

\begin{tabular}{|c|c|c|c|}
\hline \multirow{2}{*}{ Patient \# } & \multirow{2}{*}{ Symptoms by Code } & \multicolumn{2}{|c|}{ Symptoms Gone } \\
\hline & & Partial by Day & Completely by Day \\
\hline 1 & RNC, LS, Hf & 3 & 3 \\
\hline 2 & RNC, C, F, Hf & 6 & 8 \\
\hline 3 & H, Hf, LS, P & 5 & 5 \\
\hline 4 & LS, P, Hf & 1 & 4 \\
\hline 5 & Hf, LS, B & 12 & 12 \\
\hline 6 & Hf, C, RNC, H, P & 8 & 10 \\
\hline 7 & L, LS, Hf & 5 & 10 \\
\hline 8 & Hf, P, H, C, RNC & 3 & 6 \\
\hline 9 & Hf, LS & 1 & 1 \\
\hline 10 & Hf, H, F, P & 1 & 5 \\
\hline 11 & $\mathrm{~B}, \mathrm{Hf}, \mathrm{H}, \mathrm{RNC}$ & 7 & 9 \\
\hline 12 & Hf, LS & 3 & 3 \\
\hline 13 & Hf, RNC, H, LS, L & 1 & 4 \\
\hline 14 & Hf, F, LS & 2 & 5 \\
\hline \multicolumn{2}{|c|}{ Average } & 4.9 Days & 6.1 Days \\
\hline
\end{tabular}

Symptomology was monitored using a severity scale of 0-9. Partial or complete elimination of symptoms was determined accordingly. Complete removal was indicated by a score of 0 . Symptoms by Code: $B=$ breathing; $L S=l o s s$ of smell/taste; $L=l u n g$ issues; $P=$ pain $\&$ body aches; $\mathrm{Hf}=$ high fever; $\mathrm{RNC}=$ runny nose and congestion; $\mathrm{C}=$ coughing; $\mathrm{F}=$ fatigue and brain fog; $\mathrm{H}=$ headaches. ${ }^{*} \mathrm{p}<0.0001$ extremely significant difference.

\section{COVID-19 Observations}

All participants recovered $100 \%$ from the COVID-19 symptoms in an average of 6.1 days compared to the 10-20 days projected by CDC for normal recovery from mild COVID-19 infections 1 and a 3-6-week recovery for severe cases [31]. In this study, $29 \%$ of participants started showing recovery to COVID-19 symptoms

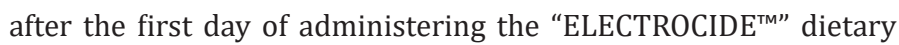
supplement and 36\% of the COVID-19 participants were symptomfree by day 4. On average COVID-19 symptoms were entirely absent by day 6. Statistically, this recovery rate is extremely significant $(\mathrm{p}<0.0001)$. By the end of the study, all COVID-19 positive individuals were retested and shown to be COVID-19 negative.

\section{Discussion}

During this study, an overall participant enthusiasm increased daily in their celebration of emerging wellness. After 7 days of treatment, it was clear that all participants achieved some new wellness standard from taking "ELECTROCIDE ${ }^{\mathrm{TM}}$," and nearly all requested to continue the program after the end of the study. In addition, there were no negative interactions or side effects reported from taking the dietary supplements from any of the participants. The present study clearly demonstrates the clinical benefits of

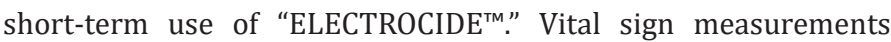
in Table 3 indicate a change toward normal body temperatures associated with wellness, where a statistically significant increase in body temperature was seen between days 1 and 6 . Increased body temperature correlates with increased performance in neurocognitive activity [32]. Similarly, there was an overall increase in blood oxygen levels which reached statistical significance by day 4 (although only data for day 6 are presented). Furthermore, participants in Groups 1 and 2 showed a statistically significant improvement in focus, sleep, and energy after "ELECTROCIDE ${ }^{\mathrm{TM}}$ " administration.

On the surface, it appears remarkable that a simple mineral solution could have such rapid and profound effects on human physiology. However, numerous studies indicate that common minerals found in dietary supplements, including "ELECTROCIDE ${ }^{\text {тм, ," }}$ have beneficial effects. For example, magnesium is a cofactor for more than 300 enzymes in the body and is involved with numerous 
functions in the brain and muscles [33]. Calcium homeostasis is critical for the function of the skeletal system, regulation of hormonal secretion, transmission of nerve impulses, and a variety of vascular activities [34]. Sodium and potassium lower blood pressure, lower cholesterol, and prevent cardiovascular disease and stroke [35]. Trace minerals like $\mathrm{Zn}, \mathrm{Fe}, \mathrm{Mn}, \mathrm{Cr}$, and $\mathrm{Cu}$ are cofactors for various enzymes, and their role in health and disease has recently been reviewed [36]. In addition to these chemically based effects, DC microcurrents present in the aqueous electroceutical product used in the current study act synergistically with the minerals $[6,7]$ to enhance its efficacy.

\section{A Feasible Mechanism of Action}

The "ELECTROCIDE ${ }^{\mathrm{TM} \text { " }}$ product used in the present study is an aqueous form of electroceuticals, where micro-electric currents are generated between the metallic ions and the salts in the solution. Electroceuticals with microfluidic properties [37] have been generated using hydrogels embedded with electrode components, including metals and semiconductors [38]. The first application of electroceutical hydrogels was developed with nanoparticles of Ag and shown to generate 100uamps DC current and to be bactericidal with only one minute of exposure [39]. To our knowledge, "ELECTROCIDE ${ }^{\mathrm{TM} \text { " }}$ is the first application of an aqueous electroceutical. We propose that the most likely mechanism of action of "ELECTROCIDE ${ }^{\text {TM" }}$ is by effecting bioelectric communication between non-neuronal cells and modulating their ion channels as previously stated [12].

It is further postulated that the "ELECTROCIDE ${ }^{\text {TM" }}$ product primarily affects squamous epithelial cells of the basal mucosa lining the inside of the mouth, where contact is first made when the product is ingested. In addition to the direct effects of ionic metals and salts on buccal epithelial cells, it is likely that the weak Electromagnetic (EM) fields generated from moving ions in the "ELECTROCIDE" ${ }^{\text {TM" }}$ solution can also affect these mucosal epithelial cells. Indeed, weak EM fields have been shown to affect membrane permeability in cheek epithelial cells [40]. Furthermore, it seems reasonable that metallic, ionic solutions can also increase the absorption of vitamins and other nutrients across the plasma membrane [36]. Thus, the health benefits observed in the present study could also be mediated by the enhanced delivery of nutrients and supplements consumed by the subjects in the study [41-55].

\section{Conclusion}

The present study demonstrated the clinical benefits of short-

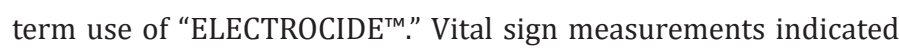
a significant change toward normal body temperatures and overall increase in blood oxygen levels. In addition, several other wellness parameters changed, including improvements in energy, focus, and sleeping. Additional benefits were seen in daily surveys for decreasing depression and anxiety, increasing happiness, improving breathing (where a difficulty was initially observed), reducing congestion, and decreasing chronic pain. Assessment of medical histories and additional notated conditions were lessened in severity for asthma, heart and lung conditions, various pains, stomach conditions, headaches, and skin conditions. There were no side effects noted during the study. The results from this study, and the preliminary observations from previous unpublished reports, indicate that there are many positive benefits towards wellness that can be gained from "ELECTROCIDE ${ }^{\mathrm{TM}}$."

\section{Acknowledgements}

Without the tremendous support of the Women of The Lovelady Center in their interest and participation this wellness study would not have been possible. I would especially like to thank Brenda Spahn, Melinda MeGahee, Joseph MeGahee, Wendy D. Kennedy, Jada Graham, Richmond Flowers, Jr. (who had the idea of this study), Brantley Kemp, and the many other supporters of this project who all believe in helping others' well-being and wellness raises the community as well.

\section{Author Contributions}

All authors researched the data for the article, contributed to the discussion of the content equally, and contributed to the writing of the article. All authors also provided equal contribution to review the manuscript before submission.

\section{Conflict of Interest}

There were no conflicts of interested noted by any of the participants relative to their roles in the study.

\section{References}

1. (2021) Centers for Disease Control. Interim Guidance on Ending Isolation and Precautions for Adults with COVID-19.

2. Horn CC, Ardell J, Fisher L, (2019) Electroceutical Targeting of the Autonomic Nervous System. Physiology 34(2): 150-162.

3. Singh M, Webster RD, Steele TWJ (2019) Voltaglue Electroceutical Adhesive Patches for Localized Voltage Stimulation. ACS Applied Biomaterials 2(6): 2633-2642.

4. Sen A, Dolly Khona, Subhadip Ghatak, Vinoj Gopalakrishnan, Kenneth Cornetta, et al. (2020) Electroceutical Fabric Lowers Zeta Potential and Eradicates Coronavirus Infectivity upon Contact.

5. Sen CK, Shomita S, Amitava D, Vishnu B, Sashwati Roy (2020) Electroceutical Management of Bacterial Biofilms and Surgical Infection. Antioxidants \& Redox Signaling 33(10): 713-724.

6. Davis CP, Weinberg S, Anderson MD, Rao G, Warren MM (1989) Effects of Microamperage, Medium, and Bacterial Concentration on Iontophoretic Killing of Bacteria in Fluid. Antimicrob Agents Chemother 33(4): 442447. 
7. Berger TJ, Spadaro JA, Bierman R, Chapin SE, Becker RO (1976) Antifungal Properties of Electrically Generated Metallic Ions. Antimicrob Agents Chemother 10(5): 856-860.

8. Del Pozo JL, Rouse MS, Mandrekar JN, Steckelberg JM, Patel R (2009) The electricidal effect: reduction of Staphylococcus and pseudomonas biofilms by prolonged exposure to low-intensity electrical current. Antimicrob Agents Chemother 53(1): 41-45.

9. Fact Sheets on Trace Minerals as found in Solution4USA formulation and actions.

10. Chen Bingdi, Wenjun Le, Yilong Wang, Zhuoquan Li, Dong Wang, et al. (2016) Targeting Negative Surface Charges of Cancer Cells by Multifunctional Nanoprobes. Theranostics 6(11): 1887-1898.

11. Fröhlich E (2012) The Role of Surface Charge in Cellular Uptake and Cytotoxicity of Medical Nanoparticles. Int J Nanomed 7: 5577-5591.

12. Famm K, Litt B, Tracey KJ, Boyden ES, Slaoui M (2013) Drug discovery: A Jump-Start for Electroceuticals. Nature 496(7444): 159-161.

13. Sonnenschein L (2013) Understanding Cellular Metabolism: Nutrition, health, and beauty. Charleston: Create Space Independent Publishing Platform.

14. Kim H, Soon Park, Greggory H, Vanessa M, Sue Cross, et al. (2016) An Overview of the Efficacy of a Next Generation Electroceutical Wound Care Device. Mil Med 181(5S): 184-190.

15. Sorgnard R (2006) Electroceutical Medicine: A New Frontier in the Treatment of Pain. American Society of Sensory Medicine 4th Annual Meeting, Newport Beach, CA.

16. Guest JF, Fuller GW, Vowden P (2017) Venous Leg Ulcer Management in Clinical Practice in the UK: Costs and Outcomes. Int Wound J 15(1): 29 37.

17. Mishra S (2017) Electroceuticals in Medicine-The Brave New Future. Indian Heart J 69(5): 685-686.

18. Vinciguerra M, Serge A, David M, Martine R, Marcelle B, et al. (2004) Extracellular Hypotonicity Increases $\mathrm{Na}$ K-ATPase Cell Surface Expression via Enhanced Na+ Influx in Cultured Renal Collecting Duct Cells. J Am Societ Nephrol 15(10): 2537-2547.

19. Puliyel JM (2003) Osmotonicity of acetoacetate: possible implications for cerebral edema in diabetic ketoacidosis. Med Sci Monit 9(4): 130 133.

20. Ytteborg J, C Dohlman (1965) Corneal Edema and Intraocular Pressure. 1. Animal Experiments. Arch Ophthalmol 74: 375-381.

21. Liu HT, Tashmukhamedov BA, Inoue H, Okada Y, Sabirov RZ (2006) Roles of two types of anion channels in glutamate release from mouse astrocytes under ischemic or osmotic stress. Glia 54(5): 343-357.

22. Saari JT, Beck JS (1975) Hypotonic Hemolysis of Human Red Blood Cells: A Two-Phase Process. J Membr Biol 23(3-4): 213-226.

23. Jacobs EA, Winter PM, Alvis HJ, Small SM (1969) Hyperoxygenation Effect on Cognitive Functioning in the Aged. N Engl J Med 281(14): 753757.

24. Kato H, Jun Araki, Kentaro Doi, Shinichiro Kuno, Kahori Kinoshita, et al. (2014) Normobaric Hyperoxygenation Enhances Initial Survival, Regeneration, and Final Retention in Fat Grafting. Plast Reconstr Surg 134(5): 951-959.

25. Rasanen J, Wood DC, Debbs RH, J Cohen, S Weiner, et al. (1998) Reactivity of the Human Fetal Pulmonary Circulation to Maternal Hyperoxygenation Increases During the Second Half of Pregnancy. Circulation 97(3): $257-$ 262.

26. Hampson NB, Neal WP, Claude AP (2003) Oxygenated Water and Athletic Performance. JAMA 290(18): 2408-2409.
27. Handajani YS, Tenggara R, Suyatna FD, Surjadi C, Widjaja NT (2009) The Effect of Oxygenated Water in Diabetes Mellitus. Med J Indonesia pp102.

28. Gruber R, Axmann S, Schoenberg MH (2005) The Influence of Oxygenated Water on the Immune Status, Liver Enzymes, and the Generation of Oxygen Radicals: A Prospective, Randomized, Blinded Clinical Study. Clin Nutr 24(3): 407-414.

29. Hyvärinen J, Laakso M, Sippel H, Roine R, Huopaniemi T, et al. (1978) Alcohol Detoxification Accelerated by Oxygenated Drinking Water. Life Sci 22(7): 553-559.

30. Fang C, Cheng-Chia T, Yan-Jye S, Chun-Ting Y, Keng-Yuan L, et al. (2020) Effects of Highly Oxygenated Water in a Hyperuricemia Rat Model. J Healthc Eng pp1-8.

31. (2021) Centers for Disease Control. Post-COVID Conditions.

32. Wright KP, Hull JT, Czeisler CA (2002) Relationship between Alertness, Performance, and Body Temperature in Humans. Am J Physiol Regul Integr Comp Physiol 283(6): R1370-1377.

33. Faryadi Q (2012) The Magnificent Effect of Magnesium to Human Health: A Critical Review. International Journal of Applied Science and Technology $2: 3$.

34. Reinwald S, Weaver CM, Kester JJ (2008) The Health Benefits of Calcium Citrate Malate: A Review of the Supporting Science. Adv Food Nutr Res 54: 219-346.

35. Whelton PK, He J (2014) Health Effects of Sodium and Potassium in Humans. Curr Opin Lipidol 25(1): 75-79.

36. Sousa C, Moutinho C, Vinha AF, Matos C (2019) Trace Minerals in Human Health: Iron, Zinc, Copper, Manganese and Fluorine. Internationa Journal of Science and Research Methodology 13: 3.

37. Park S, Yuanyuan Guo, Xiaoting Jia, Han Kyoung C, Benjamin Grena, et al. (2017) One-Step Optogenetics with Multifunctional Flexible Polymer Fibers. Nat neurosci 20(4): 612-619.

38. Scaini G, João Quevedo, Dawn Velligan, David LR, Henriette R, et al. (2018) Second Generation Antipsychotic-Induced Mitochondrial Alterations: Implications for Increased Risk of Metabolic Syndrome in Patients with Schizophrenia. Eur Neuropsychopharmacol 28(3): 369-380.

39. Vieira CM, Franco OH, Restrepo CG, Abel T (2020) COVID-19: The forgotten priorities of the pandemic. Maturitas 136: 38-41.

40. Shckorbatov YG, Valery GS, Valerya VN, Valentine AG, Svetlana PS, et al. (2002) Application of Intracellular Microelectrophoresis to Analysis of the Influence of the Low-Level Microwave Radiation on Electrokinetic Properties of Nuclei in Human Epithelial Cells. ELECTROPHORESIS 23(13): 2074-2079.

41. Bar-On YM, Flamholz A, Phillips R, Milo R (2020) SARS-CoV-2 (COVID-19) by the Numbers. ELife 9: e57309.

42. Eisengart JB, King KE, Shapiro, EG, Whitley CB, Muenzer J (2020) The Nature and Impact of Neurobehavioral Symptoms in Neuronopathic Hunter Syndrome. Mol Genet Metab Rep 22: 100549.

43. Farooq M A, Dietz KJ (2015) Silicon as Versatile Player in Plant and Human Biology: Overlooked and Poorly Understood. Frontiers in Plant Science 6: 994.

44. Hathout RM, Kassem DH (2020) Positively Charged Electroceutical Spun Chitosan Nanofibers Can Protect Health Care Providers From COVID-19 Infection: An Opinion. Front Bioeng Biotechnol 8: 885

45. Inoue T, Moore P, Tsai B (2011) How Viruses and Toxins Disassemble to Enter Host Cells. Annu Rev Microbiol 65(1): 287-305.

46. Lindinger Michael I (2021) Structured Water: Effects on Animals. J Anim Sci 99(5): skab063. 
47. Lopez MJ, Hall CA (2021) Physiology, Osmosis. StatPearls.

48. (2017) Loyola University Health System. Surgical site infections are the most common and costly of hospital infections: Guidelines for preventing surgical site infections are updated. ScienceDaily.

49. Carlucci PM, Tania A, Christopher P, Harish R, Simon J, et al. (2020) Hydroxychloroquine and Azithromycin plus Zinc vs Hydroxychloroquine and Azithromycin Alone: Outcomes in Hospitalized COVID-19 Patients.

50. Puertollano MA, Puertollano E, De Cienfuegos GÁ, De Pablo MA (2011) Dietary Antioxidants: Immunity and Host Defense. Curr Top Med Chem 11(14): 1752-1766.

51. Rowlands DS, Shultz SP, Ogawa T, Aoi W, Korte M (2014) The Effects of Uniquely-Processed Titanium on Biological Systems: Implications for Human Health and Performance. J Funct Biomater 5(1): 1-14.
52. Saper RB, Rebecca R (2009) Zinc: an essential micronutrient. Am Fam Physician 79(9): 768-772.

53. Shibasaki K, Suzuki M, Mizuno A, Tominaga M (2007) Effects of Body Temperature on Neural Activity in the Hippocampus: Regulation of Resting Membrane Potentials by Transient Receptor Potential Vanilloid 4. J Neurosci 27(7): 1566-1575.

54. Sonnenschein L, Robert Weinberg, H Thomas Cotter, Lawrence A Salvo, Tiberious Etyang, et al. (2020) Historical and Current Conditions Including COVID-19 Requiring Anti-Pathogenic Surveillance and Proper Immunogenic Support. Am J Biomed Sci Res 8(4): 309-312.

55. Sonnenschein L (1999) Method of stimulating growth in aquatic animals using growth hormones. European patent, \# EP 1178813A1. 